\title{
Safety of Percutaneous Transhepatic Biliary Stenting in Patients with Obstructive Jaundice
}

\author{
Rafeah Khan1, Zainab Hussain2, Vaqar Bari2 and Azeikah Ben Fiaz³
}

\begin{abstract}
Objective: To determine the safety of percutaneous transhepatic biliary stenting (PTBS) in patients with obstructive jaundice.

Study Design: A case series.

Place and Duration of Study: Department of Radiology, The Aga Khan University Hospital Karachi, Pakistan, from February 2012 to April 2013.

Methodology: Patients with obstructive jaundice due to any cause referred for percutaneous transhepatic biliary stenting were included in the study. Patients were excluded if they had undergone previous ERCP, had guided transhepatic biliary stenting, previous percutaneous transhepatic biliary stenting and were lost to follow-up. Follow-up was taken at a 2, 4 and 6 week interval and clinical outcome was assessed as the difference between the bilirubin levels at base line and 6 weeks after PTBS, which was measured using Freidman's test.

Results: A total of 102 patients were included; $59(58 \%)$ were males and $43(42 \%)$ were females. The age ranged from 21 to 89 years. A total of 30 patients experienced complications making an overall complication rate of $29.4 \%$; 20 experienced minor and 10 experienced major complications. Pain was the most frequent minor complication ( $\mathrm{n}=15$ patients, $14.7 \%$ ) followed by biliary leakage, fever and cholangitis. Major complications included death in 10 (10\%) patients followed by biliary peritonitis and septicemia.
\end{abstract}

Conclusion: PTBS achieved satisfactory palliation with a low complication rate in patients with obstructive jaundice.

Key Words: Percutaneous transhepatic cholangiogram, Percutaneous transhepatic biliary drainage, Percutaneous transhepatic biliary stenting, Obstructive jaundice.

\section{INTRODUCTION}

Obstructive jaundice is one of the commonest biliary tract conditions with a prevalence of $2 \%$ in the Pakistani population and is frequently encountered in the fifth and sixth decade of life. The studies show that the number of cases of biliary obstruction due to malignancy are increasing. 1,2 Cholestasis because of obstructive jaundice impairs the immune response, blood clotting, and other functions. Therefore, the objective of palliative treatment is to relieve jaundice-related symptoms, prevent cholangitis, prolong survival, and improve overall quality of life.

Molnar et al. introduced percutaneous transhepatic biliary drainage (PTBD) in 1974. ${ }^{3}$ Since then, percutaneous transhepatic biliary stenting (PTBS) is routinely used for biliary obstruction as it causes less trauma and has a higher success rate.4,5 Technical

1 Department of Radiology, Indus Hospital, Karachi, Pakistan

2 Department of Radiology, The Aga Khan University Hospital, Karachi, Pakistan

3 Department of Radiology, Norfolk and Norwich University Hospital, Colney Ln, Norwich NR4 7UY, UK

Correspondence: Dr. Zainab Hussain, Department of Radiology, The Aga Khan University Hospital, Stadium Road, Karachi, Pakistan

E-mail: zainab.hussain@aku.edu

Received: May 26, 2017; Accepted: September 05, 2018 success rate of $98 \%$ was reported by Kaskarelis et al. in treatment of biliary obstruction with stents, and Indar et al. showed an improvement in hyperbilirubinemia to normal levels in $96 \%$ patients with obstructive jaundice one month after PTBS.6,7

Liu et al. assessed the safety and effectiveness of percutaneous biliary stent placement in patients with biliary obstruction. ${ }^{8}$ They found a complication rate of $7.1 \%$ and proved that stenting of bile duct provides a safer and less invasive alternative to surgical palliation, with an acceptable clinical outcome. This was checked by examination of liver function tests and US to see the alleviation of obstructive jaundice.

Haq et al. determined the rate of complication of PTBS in 2001. Overall major complications, which included death, severe septicemia, and biliary peritonitis occurred in $23 \%$; and patients with minor complications including cholangitis, fever, bacteraemia, pain, minor bleeding or leakage were seen in $18 \%$ patients. There was a high overall complication rate of $41 \% .^{9}$

As the complication rate differed so greatly from Western studies, it was decided to evaluate the safety of PTBS in palliative treatment of obstructive jaundice to have some clarity as to why the rates differ so greatly. This study will provide the current magnitude of complications due to PTBS in the local patients.

The objective of the study was to determine the safety of PTBS in patients with obstructive jaundice. 


\section{METHODOLOGY}

The study was carried out in Department of Radiology, The Aga Khan University Hospital, Karachi, from February 2102 to April 2013. This was a descriptive study with non-probability, purposive sampling technique.

The sample size was calculated on WHO software version of sample size determination in health studies using outcome variable of safety. Taking the lowest complication rate of PTBS, reported as $7.1 \%$ with confidence interval of $95 \%$ and absolute precision of $5 \%$, the calculated sample size was 102 patients. 8 Inclusion criteria were patients of all ages and gender referred to the Radiology Department, The Aga Khan University Hospital for PTBS having obstructive jaundice. Exclusion criteria were those patients having undergone previous ERCP-guided transhepatic biliary stenting, previous percutaneous transhepatic biliary stenting, and those who were lost to follow-up.

Informed consent was taken from the patients or relatives and the procedure was performed under sedation on Siemens Artis Axiom angiography machine by an experienced radiologist with minimum five years' experience. Absence of minor or major complications was assessed by clinical follow-up and laboratory investigations after 2, 4 and 6 weeks' interval. Final outcome was measured at the end of six weeks.

Patients' data were collected using a proforma customised. Data were entered and analysed by using SPSS statistical package version 19 software. Data were checked for normality using the Shapiro-Wilk test. Associations were checked between extremes of age, multiple comorbids and severity of disease using the Chi-square test. Stratification of age, comorbids and severity of disease was performed for all major complications using Fisher exact test to look for confounding effects. The $p$-value of less than or equal to 0.05 was considered significant.

Descriptive analysis was conducted, i.e. frequencies and percentages for categorical variables like gender, patency of stent, complications and cause of obstructive jaundice with median and interquartile ranges (IQR) and mean and standard deviations for continuous variables like age, serum bilirubin levels, and duration of jaundice.

The difference between the bilirubin levels at base line and 6 weeks after PTBS was used to assess the clinical outcome, which was measured using the Freidman's test.

\section{RESULTS}

A total of 102 patients were included in the study, who fulfilled the inclusion and exclusion criteria. Out of the 102 patients, $59(58 \%)$ were males and $43(42 \%)$ were females.

Patients of all ages were included with an age range of 21-89 years. The mean age was $58 \pm 15.9$ years. Age was grouped into 21-54 years and 55-89 years. Multiple comorbid conditions such as diabetes mellitus (DM), ischemic heart disease (IHD), chronic renal failure (CRF), and chronic liver parenchymal disease (CLD) were taken into consideration and severity of disease was divided into benign and malignant.

Ischemic heart disease was the only complication that was statistically significant in the older age group (Table I). No statistical significance was seen between extremes of age and benign or malignant nature of disease (0.678).

\begin{tabular}{l|c|c|c|c} 
Table I: Stratification of age and multiple comorbids. \\
\hline Comorbid condition & $\begin{array}{c}\text { Age } \\
21-54 \text { years }\end{array}$ & $\begin{array}{c}\text { Age } \\
55-89 \text { years }\end{array}$ & Chi-sq & p-value \\
\hline Diabetes & $21(45.6 \%)$ & $18(28.5 \%)$ & 1.95 & 0.16 \\
Chonic renal failure & $10(21.7 \%)$ & $14(25 \%)$ & 0.14 & 0.69 \\
Ischemic heart disease & $10(26 \%)$ & $22(35.7 \%)$ & 3.61 & $0.05^{*}$ \\
Chronic liver disease & $4(10.8 \%)$ & $10(16 \%)$ & 1.79 & 0.181 \\
\hline
\end{tabular}

${ }^{*} P$ value less than or equal to 0.05 was considered as statistically significant

The $p$-value less than or equal to 0.05 was considered as statistically significant.

Final outcome of the PTBS was assessed by pre- and post-bilirubin levels. Total bilirubin was assessed at 2, 4 and 6 weeks to see the palliative effect of the treatment. Significant difference in bilirubin levels were found at baseline pre-stenting and as early as 2 weeks after the stenting from where it kept on improving till 6 weeks, which marked the end of the monitoring phase. Median bilirubin level was $15 \mathrm{mg} / \mathrm{dL}$ (IQR=3), which reduced to 6 at 2 weeks, 5 at 4 weeks and 4 at 6 weeks $(p<0.001)$.

Majority of the causes of obstructive jaundice were secondary to cholangiocarcinoma (Cholangio CA) with a total of 34 patients (33.3), followed by carcinoma of pancreas (CA pancreas) and carcinoma of gall bladder with a total of $32(31.3 \%)$ and $21(20.5 \%)$ patients, respectively. Ten patients had obstructive jaundice secondary to metastases from primary diseases including hemangioendothelioma, synovial carcinoma, prostatic carcinoma, colorectal carcinoma, adenocarcinoma of appendix and breast carcinoma.

A total of 30 patients experienced complications making an overall complication rate of $29.4 \%$. Out of these, 30 patients, $20(66.7 \%)$ experienced minor and 10 (33.3\%) experienced major complications.

Analysis of minor complications showed pain as the most frequent minor complication with a total of 15 patients $(14.7 \%)$ followed by biliary leakage, fever and cholangitis with a total of $10(10 \%), 7(7 \%)$ and $4(4 \%)$ patients, respectively (Figure 1).

Analysis of major complications showed outcome of death in $10(10 \%)$ patients followed by biliary peritonitis and septicemia in $6(6 \%)$ and $6(6 \%)$ patients, respectively.

No significant confounding of age, severity and comorbids was noted with major complications (Table II). 


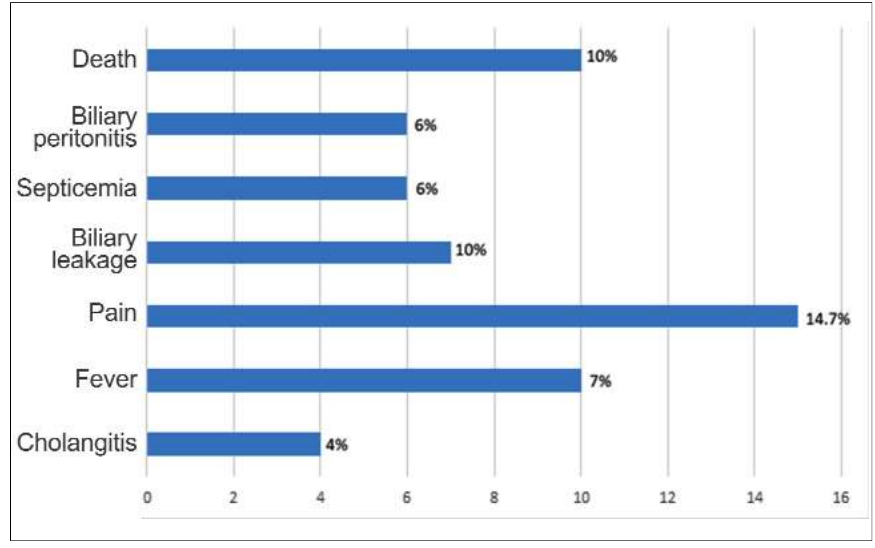

Figure 1: Minor and major complications secondary to PTC

Table II: Association of risk factors with major complications.

\begin{tabular}{l|c|c|c|c}
\hline & Death & Septicemia & BIL pritonitis & p-value \\
\hline Age & 0.108 & 1.198 & 1.198 & $>0.05$ \\
Severity & 0.336 & 0.193 & 0.193 & $>0.05$ \\
DM & 3.74 & 1.25 & 1.25 & $>0.05$ \\
CRF & 1.128 & 0.167 & 0.167 & $>0.05$ \\
IHD & 2.35 & 0640 & 0.640 & $>0.05$ \\
CLD & 0.130 & 0.047 & 0.047 & $>0.05$ \\
\hline
\end{tabular}

\section{DISCUSSION}

Biliary obstruction resulting in obstructive jaundice is increasing in our population. Although clinical presentation and laboratory work up is the mainstay of diagnosis, radiological investigations, especially ultrasound and subsequently PTC, also play a role.

Surgical intervention, whether curative or palliative, is the first line of treatment; however, often enough patients presenting with advanced stages of malignancies are poor surgical candidates due to the high risk of complications. The operative mortality may be as high as 2-5\%; and in such circumstances ERCP, PTBD and PTBS are then used for palliative treatment to improve the quality of life and extend the survival time. ${ }^{7}$

In this study, a total of 102 patients were included according to the inclusion and exclusion criteria. Successful stenting was performed in all patients with adequate post-stenting biliary drainage (Figure 2). They had a mean age of $58 \pm 15.9$ years with minimum age of 21 years and maximum age of 89 years.

Out of the 102 patients, majority were males $(n=59$, $58 \%$ ). This is a contradiction to other studies which shows the incidence of biliary disease to be higher in females. ${ }^{10-13}$ This discrepancy could be due to the fact that none of the patients who presented to this Department had cholelithiasis, which is much more common in women.

No statistical significance was seen between age, multiple comorbids and severity of disease. Most of the patients had jaundice for one month and all malignancies were advanced and inoperable at the time of presentation.

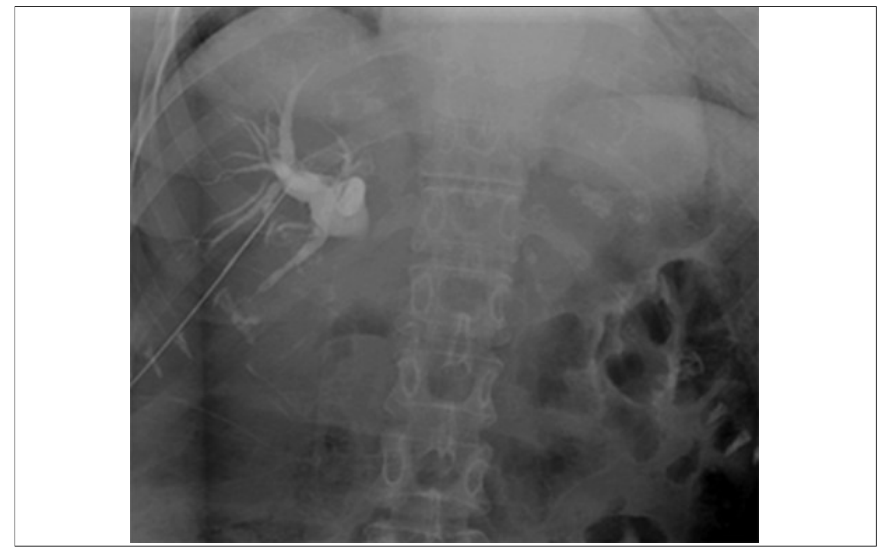

Figure 2a: Preprocedure percutaneous transcholangiogram reveals proximal CBD dilatation with intrahepatic dilatation of the biliary channels.

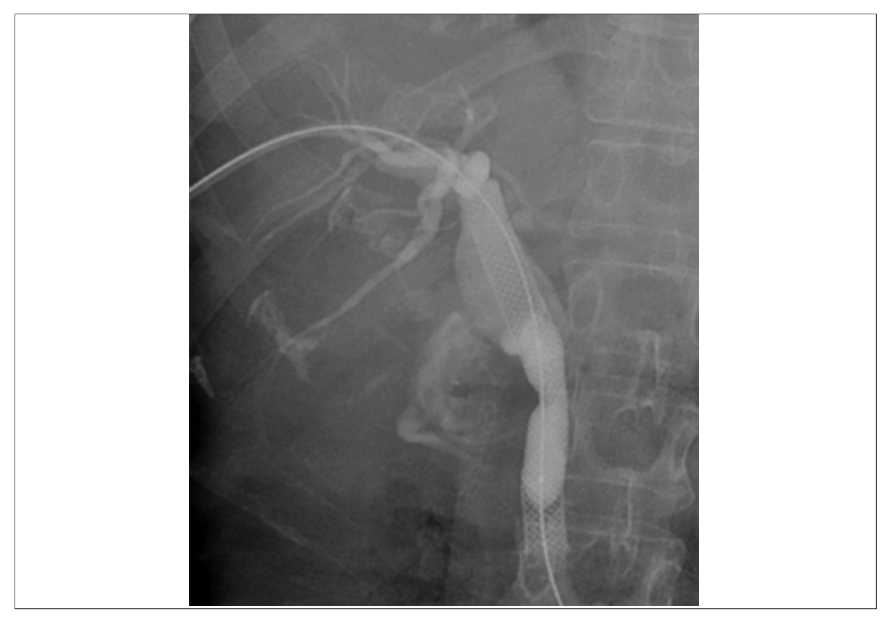

Figure 2b: Postprocedural placing of biliary stent with significant drainage of the contrast in the duodenum below depicting complete patency of the stent.

Two out of the three patients with benign obstructive jaundice in this study were in younger age group, while most of the malignant causes were in the elder age group ( $n=69,67.6 \%)$. This is comparable with the previous studies which show the incidence of malignant obstructive jaundice to be higher in patients of older age group. ${ }^{11-13}$

The total bilirubin was assessed at the time of presentation, 2, 4 and 6 weeks, and it showed a maximum decline of $50 \%$ in 2 weeks, which overall improved the quality of life. This is in concordance with previous study by Haq et al. ${ }^{9}$ After 2 weeks, the decline in serum bilirubin levels were more gradual and in 19 patients, the levels actually increased after 4 weeks (Figure 1).

This study showed that bulk of the cases with obstructive jaundice were secondary to malignant diseases $(97 \%)$, which is in concordance with previous studies.11-16 Only 3 out of the 102 patients presented with benign biliary strictures.

Out of all the malignancies, the commonest one in our study was cholangio $C A(n=34,33.3 \%)$, followed by $C A$ 
Annex 1: Patient Proforma: Safety and efficacy of percutaneous transhepatic biliary stenting in obstructive jaundice.

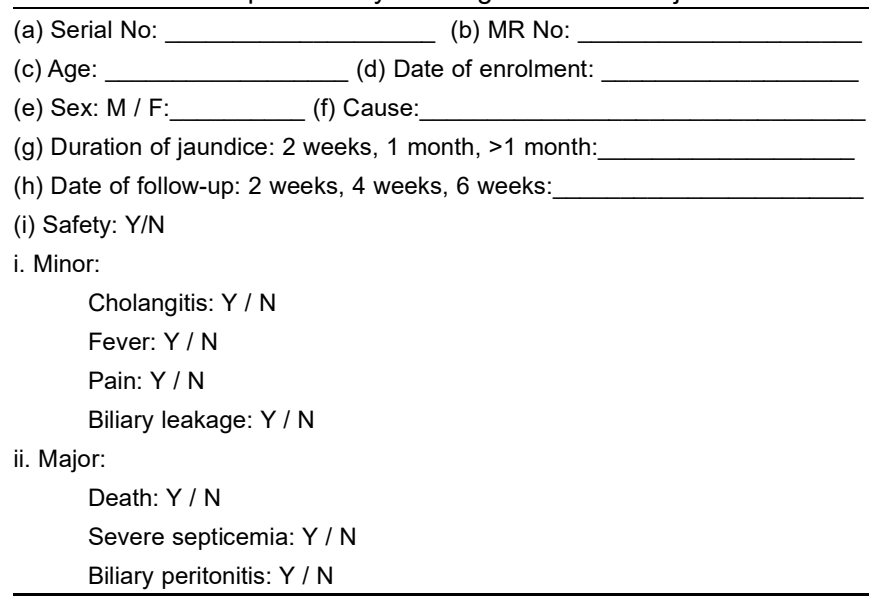

pancreas $(n=32,31.3 \%)$, and CA gall bladder $(n=21$, $20.5 \%$ ). Previous studies show pancreatic carcinoma to be the leading cause of biliary obstruction. ${ }^{11-16} \mathrm{~A}$ study performed by Sharma et al. showed CA gall bladder to be the leading cause of obstructive jaundice. ${ }^{11}$

The overall complication rate was $29.4 \%$ which is higher than the previously reported $7 \%$ by Liu et al. ${ }^{8}$ Previously, in the local population the overall complication rate was reported as $41 \%$ by $\mathrm{Haq}$ et al. which was markedly higher than the present figure. That could be due to the small sample size in the earlier study. ${ }^{9}$ Another reason may be increased expertise with the procedure as both studies were conducted in the same Department.

In this study, minor complications were seen in $19.6 \%$ of the patients in comparison to $23 \%$ seen in previous study. Pain was the most common complaint comprising $14.7 \%$ of the minor complication; whereas, cholangitis was the most common minor complication in a previous study. 8

Severe septicemia leading to eventual death was the major complication seen in a previous study; however, in this study death was the commonest major complication. Six cases were secondary to septicemia. The total major complication rate in this study was $9.8 \%$.

Controversies remain about whether palliative surgery is more beneficial in comparison with percutaneous biliary procedures. A study by Niemela et al. showed that patients with cancer who underwent PTBD for biliary obstruction had a poor median overall survival. ${ }^{17}$ Another study by Pranculis showed that PTBS with uncovered self expandable metallic stent is an effective and safe method for palliative treatment of malignant biliary obstruction. ${ }^{18}$ Overall, the results are somewhat in contrast with the international data and further work should be done to fully discern, if the palliative effect of PTBS outweighs the risk of complication.

Larger scale prospective studies of greater duration should be conducted to fully evaluate the safety. Another limitation was the inability to evaluate if death in four patients was secondary to procedural complication or if it was secondary to the advanced stage of the disease. However, due to detailed analysis of confounding, this factor was significantly eliminated.

The authors suggest further studies be conducted and quality of life be assessed alongside the complication rate with a more substantial emphasis on sub-group analysis, so that we may be able to assess if the palliative effect of this procedure is worth the controversial complication rate.

\section{CONCLUSION}

PTBS is a good procedure for palliative treatment of obstructive jaundice and should be used in patients who are poor surgical candidates and those in whom ERCP has failed. Although the complication rate in this study is higher than in international literature, there does appear to be a declining trend at the same institute. Pain was the commonest minor complication and death was the commonest major complication.

Disclosure: This article is based on the dissertation written by Dr. Rafeah Khan as partial requirement for FCPS.

\section{REFERENCES}

1. Ahmad N, Aamir AH, Hussain I, Ghulam S. Annual prevalence of various diseases in hospitalized patients in a tertiary level teaching at Peshawar. Pak J Med Res 2004; 43:166-71.

2. Gupta AK, Singh A, Goel S, Tank R. Profile and pattern of obstructive jaundice cases from a tertiary care teaching hospital of Uttar Pradesh. Int Surg J 2017; 4:743-6.

3. Molnar W, Stockum AE. Relief of obstructive jaundice through percutaneous transhepatic catheter - a new therapeutic method. Am J Roentgenol Radium Ther Nucl Med 1974; 122:356-67.

4. Garcarek J, Kurcz J, Guzinski M, Janczak D, Sasiadek M. Ten years single center experience in percutaneous transhepatic decompression of biliary tree in patients with malignant obstructive jaundice. Adv Clin Exp Med 2012; 21:621-32.

5. Guo YX, Li YH, Chen Y, Chen PY, Luo PF, Li Y, et al. Percutaneous transhepatic metal versus plastic biliary stent in treating malignant biliary obstruction: a multiple center investigation. Hepatobiliary Pancreat Dis Int 2003; 2:594-7.

6. Kaskarelis IS, Papadaki MG, Papageorgiou GN, Limniati MD, Malliaraki NE, Piperopoulos PN. Long term follow-up in patients with malignant biliary obstruction after percutaneous placement of uncovered wall stent endoprostheses. Acta Radiol 1999; 40:528-33.

7. Indar AA, Lobo DN, Gilliam AD, Gregson R, Davidson I, Whittaker S, et al. Percutaneous biliary metal wall stenting in malignant obstructive jaundice. Eur J Gastroenterol Hepatol 2003; $15: 915-9$.

8. Liu F, Zhang CQ, Wang GC, Liu FL, Xu HW, Hu L, et al. Percutaneous biliary stent placement in palliation of malignant bile duct obstruction. Gastroenterol Res 2009; 2:289-94.

9. Haq TU, Sanaullah M, Mohsin H, Sheikh MY, Ahmed B. Percutaneous transhepatic biliary stenting. J Pak Med Assoc 2001; 51:308-12. 
10. Mohamed S, Syed AL. Management of obstructive jaundice: Experience in a tertiary care surgical unit. Pak J Surg 2007; 23:23-5

11. Gracanin AG, Kujundzic M, Petrovecki M, Romic Z, Rahelic D. Etiology and epidemiology of obstructive jaundice in continental Croatia. Coll Antropol 2013; 37:131-3.

12. Sharma MP, Ahuja V. Aetiological spectrum of obstructive jaundice and the diagnostic ability of ultrasonography: A clinician's perspective. Trop Gastroenterol 1999; 20:167-9.

13. Syed N, Mohammad SA, Umair UI, Shafiq UR. Etiological spectrum of obstructive jaundice. Med Channel 2010; 16: 299-301.

14. Khurram S, Qasim A, Shirin M, Aiza J, Aisha E, Sarmad L, et al. Evaluation of the aetiological spectrum of obstructive jaundice. J Ayub Med Coll Abbottabad 2008, 20:62-6.
15. Lawal D, Oluwole S, Makanjuola D, Adekunle M. Diagnosis, management and prognosis of obstructive jaundice in lle-lfe, Nigeria. West Afr J Med 1998; 17:255-60.

16. Mabula JB, Gilyoma JM, Mchembe MD, Jaka H, Kamugisha E, Kidenya $\mathrm{B}$, et al. Predictors of outcome among patients with obstructive jaundice at Bugando Medical Centre in north-western Tanzania. Tanzan J Health Res Oct 2013; 15: 216-22.

17. Niemelä J, Kallio R, Ohtonen P, Perälä J, Saarnio J, Syrjälä H. Is palliative percutaneous drainage for malignant biliary obstruction useful? World J Surg 2018; 42:2980-6.

18. Pranculis A, Kievišas M, Kievišiene L, Vaicius A, Vanagas $T$, Kaupas RS, et al. Percutaneous transhepatic biliary stenting with uncovered self-expandable metallic stents in patients with malignant biliary obstruction - Efficacy and survival analysis. Pol J Radiol 2017; 82:431-40. 